\title{
Wilms' tumor in a 51-year-old patient: An extremely rare case and review of the literature
}

\author{
JIA HU ${ }^{1-3}$, LU JIN $^{1,3}$, TAO HE $^{1-3}$, YIFAN LI $^{1,3}$, YANG ZHAO $^{1}$, YU DING $^{1}$, XIANXIN LI $^{1}$, YUNCHU LIU $^{1}$, \\ YAOTING GUI ${ }^{3}$, XIANGMING MAO ${ }^{1}$, YONGQING LAI ${ }^{1,3}$ and LIANGCHAO NI ${ }^{1,3}$ \\ ${ }^{1}$ Department of Urology, Peking University Shenzhen Hospital, Shenzhen, Guangdong 518036; \\ ${ }^{2}$ Guangzhou Medical University, Guangzhou, Guangdong 510182; ${ }^{3}$ Guangdong Key Laboratory \\ of Male Reproductive Medicine and Genetics, Peking University Shenzhen Hospital, \\ Shenzhen PKU-HKUST Medical Center, Shenzhen, Guangdong 518036, P.R. China
}

Received November 5, 2015; Accepted February 22, 2016

DOI: $10.3892 / \mathrm{mco} .2016 .839$

\begin{abstract}
Wilms' tumor or nephroblastoma is a common kidney malignant tumor in childhood, accounting for $\sim 5 \%$ of all pediatric tumors. At present, reports on Wilms' tumor occurring in adults, particularly at ages $>30$ years, are extremely rare. The majority of the cases of adult Wilms' tumor are closely associated with chemotherapy. Furthermore, in rare cases, Wilms' tumor is characterized by three classic types of cells, namely blastemal, stromal and epithelial cells. We herein report a case of Wilms' tumor with three classic types of cells on histological examination in a 51 year-old male patient who had received prior chemotherapy. The patient promptly underwent radical nephrectomy and remains alive. A review of previously presented cases of adult Wilms' tumor from PubMed database was also performed.
\end{abstract}

\section{Introduction}

Wilms' tumor or nephroblastoma, named by the German surgeon Carl Max Wilhelm Wilms' in the 19th century, is the second most common intra-abdominal tumor in young children, with a peak incidence between 2 and 5 years. The incidence of Wilms' tumor in adults is extremely low, and the total cases presented to date account for $<1 \%$ (1). However, the precise number of cases of Wilms' tumor in adults remains unknown, as a significant number of cases are either insufficiently documented or misdiagnosed. The stage-for-stage prognosis in adults is poorer compared with that in children. Due to the improved practicable treatments, the survival of

Correspondence to: Professor Liangchao Ni or Professor Yongqing Lai, Department of Urology, Peking University Shenzhen Hospital, 1120 Lianhua Road, Shenzhen, Guangdong 518036, P.R. China

E-mail: 13609618222@163.com

E-mail: yqlord@163.com

${ }^{*}$ Contributed equally

Key words: adult Wilms' tumor, renal, Wilms' tumor, oncogene adult patients has improved significantly from $<30$ to $>90 \%$ (2). To the best of our knowledge, 9 cases of adult Wilms' tumor were previously published (Table I) and our patient, aged 51 years, is the second oldest patient reported to date. A radical nephrectomy was performed with subsequent chemotherapy. The present study was approved by the Ethics Committee of Peking University Shenzhen Hospital (Shenzhen, China) and written informed consent was obtained from the patient regarding the publication of his clinicopathological data.

\section{Case report}

A 51 year-old man was diagnosed with a left kidney tumor on routine examination and was admitted to the Department of Urology of Peking University Shenzhen Hospital (Shenzhen, China) for further evaluation. The patient had no urinary or respiratory symptoms and had not undergone previous surgery. The general examination revealed no significant findings. The patient had a heart rate of 88 beats $/ \mathrm{min}$, a blood pressure of $118 / 67 \mathrm{mmHg}$, a temperature of $36.8^{\circ} \mathrm{C}$ and a respiratory rate of 22 breaths/min.

Laboratory examination revealed a haemoglobin level of $11.3 \mathrm{~g} / \mathrm{dl}$, and a white blood cell count of $6.86 \times 10^{9} / 1$, with $59.8 \%$ granulocytes. The glucose level was $4.98 \mathrm{mmol} / \mathrm{l}$, the blood urea nitrogen was $7.95 \mathrm{mmol} / \mathrm{l}$ and the serum creatinine was $79.2 \mu \mathrm{mol} / 1$. The liver function tests and serum electrolyte levels were normal. Urine examination revealed several erythrocytes per high-power field. The chest X-ray, renal function tests, cardiovascular and neurological investigations were largely normal. The non-contrast computed tomography (CT) scan of the kidneys revealed a $4.0 \times 4.0 \times 4.5-\mathrm{cm}$ round hypodense mass [39 Hounsfield units (HU)] arising from the upper pole of the left kidney (Fig. 1A). The contrast-enhanced CT revealed a heterogeneously enhanced lesion (51 HU) (Fig. 1B).

The patient underwent left radical nephrectomy and macroscopically the tumor was a well-encapsulated mass that did not appear to invade neighboring tissue. The microscopic examination identified blastemal cells, undifferentiated or differentiated to various degrees, and epithelial and stromal lineages in different proportions (Fig. 2A). However, the most typical characteristic was the presence of undifferentiated 


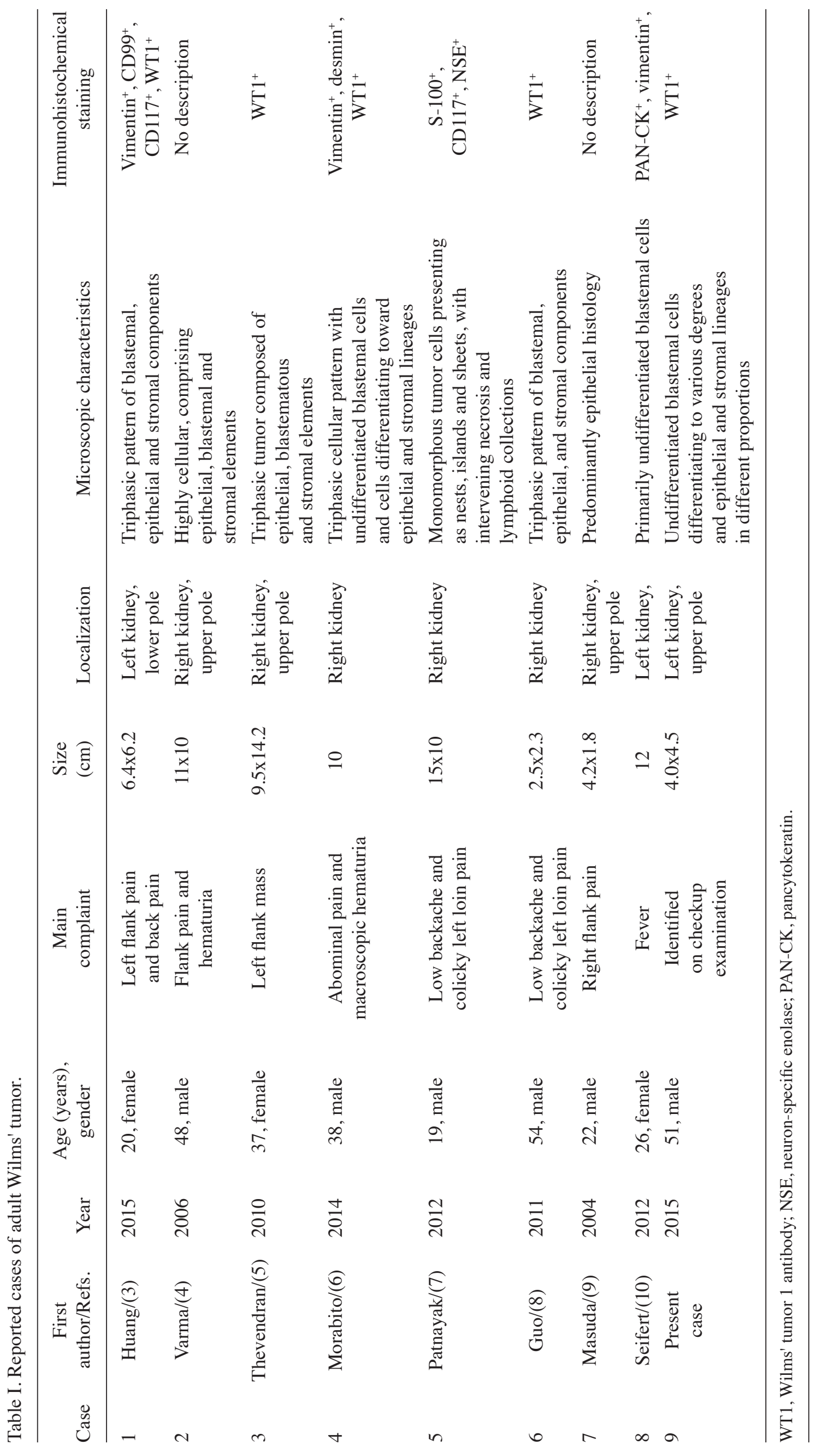



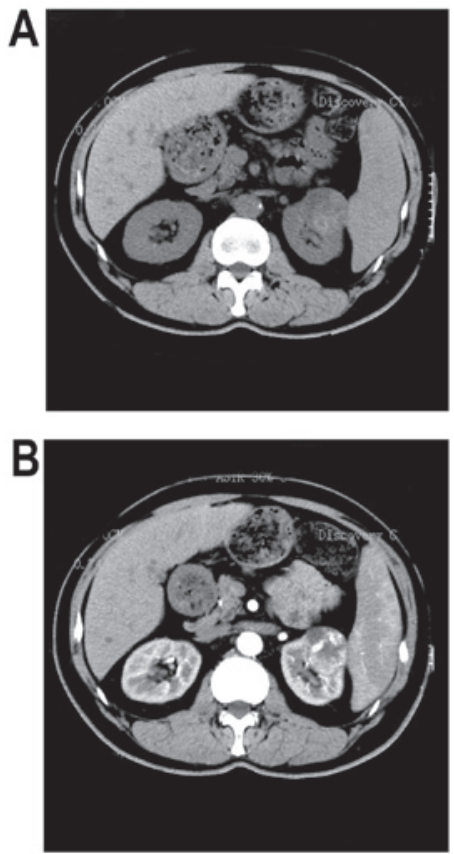

Figure 1. Renal computed tomography (CT) scan. (A) A round hypodense mass $(39 \mathrm{HU})$, sized $4.0 \times 4.0 \times 4.5-\mathrm{cm}$ was identified in the upper pole of the left kidney (non-contrast CT). (B) The mass was heterogeneously enhanced (51 HU) on contrast-enhanced CT.
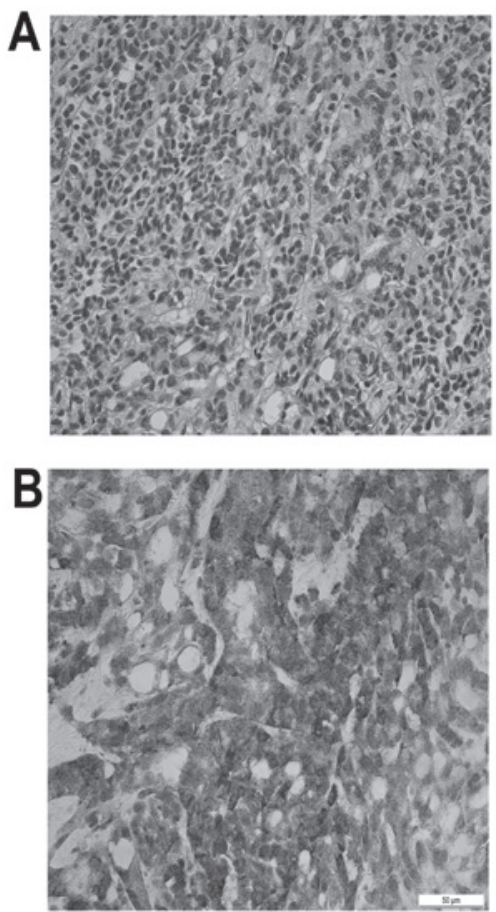

Figure 2. Results of pathological and immunohistochemical examination. (A) Blastemal cells undifferentiated and differentiated to various degrees, with different proportions of epithelial and stromal lineages (magnification, $\mathrm{x} 400$ ). (B) Positive immunohistochemical staining with Wilms' tumor 1 antibody (magnification, $\mathrm{x} 400$ ).

blastemal cells. On immunohistochemical staining, Wilms' tumor 1 antibody (WT1) was found to be positive (Fig. 2B) and the renal neoplasm was confirmed as Wilms' tumor. Positron emission tomography-CT revealed no metastases. Chemotherapy was performed regularly and there was no evidence of cancer on medical examination at the 2-year follow-up (11).

\section{Discussion}

Nephroblastoma, also referred to as Wilms' tumor, is a embryonal neoplasm originating from nephrogenic blastemal cells, which replicates the histology of the kidneys and usually exhibits various patterns of differentiation (12). Approximately one in every 7,000 children suffer from this disease (12). There are no gender differences and Wilms' tumor occurs in equal frequency in both kidneys. The mean age is 35 and 45 months for males and females, respectively, and $98 \%$ of the cases occur at ages $<10$ years (12). Wilms' tumor has also been reported in adults, but it is extremely rare (13). The most common main complaints are local pain and painless hematuria, but in children a palpable mass is more common (14). The tumor is often detected by abdominal palpation, CT and ultrasound scan (15). Compared with pediatric counterparts, adult patients with advanced clinical stage exhibit metastatic manifestations more frequently (10 vs. 29\%, respectively) (12). The prognosis of adult Wilms' tumor is worse compared with that in the pediatric population, and there is no detailed explanation for that phenomenon to date. Approximately 5\% of Wilms' tumors are associated with an unfavorable outcome and are recognized pathologically as having unfavourable histology, mainly due to the presence of nuclear anaplasia (12).

In pediatric patients as well as in adults, Wilms' tumors present as triphasic embryonic kidney tumors, and their histological appearance has the characteristics of marked structural diversity (5). Although, classic Wilms' tumor consists of three types of cells (blastemal, stromal and epithelial), the presence of all three types is uncommon in the same case (16). The presence of blastemal cells is the predominant histological component of Wilms' tumors, and it appears in distinctive patterns. Blastemal cells exhibit scant cytoplasm and are very small, mitotically active, with rotund and overlapping nuclei containing coarsely distributed chromatin and evenly small nucleoli (17). An epithelial component is present in several Wilms' tumors, and this feature may be represented by primitive structures merely recognizable as tubular formations. Other Wilms' tumors are composed of papillary and tubular elements that are easily recognized, recapitulating normal stages of nephrogenesis (18). Various stromal patterns may occur and cause diagnostic difficulties when there is lack of blastemal and epithelial differentitation. The common undifferentiated RCC has been divided into clear-cell and non-clear-cell categories, with papillary RCC forming the most common subtype of non-clear-cell RCC $(19,20)$. To the best of our knowledge, various undifferentiated tumors in adults should be considered in the differential diagnosis, particularly when the carcinoma is predominantly monophasic.

From a genetics aspect, $\sim 10 \%$ of Wilms' tumors develop in association with one of several well-characterized dysmorphic syndromes. The molecular events of adult Wilms' tumorigenesis have not been fully elucidated (21). However, similar to other tumor genes, the location of Wilms' tumor may be detected by cytogenetic analysis of DNA from the patients whose abnormalities were genetically determined, enabling the prediction of a number of chromosomal disruptions 
significantly associated with phenotypic abnormalities. The constitutional loss of band 13 of the short arm of chromosome 11 (11p13 or WT1) was significantly associated with adult Wilms' tumors (22). The deletion of genetic material from chromosome $11 \mathrm{p} 13$ was clearly associated with tumorigenesis, indicating that some critical deletions may involve the tumor suppressor genes. The candidate genes were entirely selected from the deleted regions of chromosome $11 \mathrm{p} 13$ and the target gene WT1 or 11p13 was isolated and cloned (23). The sequence analysis demonstrated that $11 \mathrm{p} 13$ acts as a transcriptional regulator whose protein product significantly affects specific DNA motifs (23). However, the accurate function of the WT1 protein remains unknown. Recent research suggests that patterns of WT1 expression may play an extremely significant role in cell differentiation of the metanephric stem, which may explain the finding of associated genitourinary abnormalities. The gene for Beckwith-Wiedemann syndrome has been localized to the chromosome 11p15 and named WT2, but the precise genetic mechanism has not yet been fully elucidated (24). Efforts to identify the precise genetic event at this locus have identified the presence of clusters of imprinted genes. At the locus, a preferential deletion of its maternal allele in several cases of Wilms' tumor reveals that genomic imprinting is associated with the pathogenesis of certain neoplasms (24). Furthermore, additional genetic loci are involved in familial Wilms' tumor in patients carrying WT1 and WT2. A proportion of patients with nephroblastoma usually have a positive family history of identical tumors. It remains unclear whether the aberration of genes results in Wilms' tumor or other tumors. Therefore, this issue requires further investigation.

There are currently no adequate treatment guidelines for adult Wilms' tumor (25). The pediatric regimen, which includes radical nephrectomy and adjuvant chemotherapy, with or without radiotherapy, is recommended for the treatment of the adult counterpart. However, there is limited information available for adult Wilms' tumor treatment if the initial chemotherapy fails or if the tumor recurs (25).

In the present case, our patient is the second oldest reported to date and he had received no prior chemotherapy. Furthermore, all three types of cells were histopathologically identified in the tumor. Therefore, we consider this to be an extremely rare case of adult Wilms' tumor.

\section{Acknowledgements}

The present study was supported by grants from the National Natural Science Foundation of China (no. 81101922), the Guangdong Province Natural Science Foundation of China (no. S2012010008365), the Science and Technology Development Fund Project of Shenzhen (nos. JCYJ20130402114702124 and JCYJ20150403091443329) and the fund of Guangdong Key Medical Subject.

\section{References}

1. Özyörük D, Demir HA, Emir S, Karakuş E and Tunç B: Occurrence of Wilms' tumor in a child with hereditary spherocytosis. Turk J Pediatr 57: 206-209, 2015.

2. Sakai K, Shimodaira S, Maejima S, et al: Dendritic cell-based immunotherapy targeting Wilms' tumor 1 in patients with recurrent malignant glioma. J Neurosurg 123: 989-97, 2015.
3. Huang JL, Liao Y, An Y and Qiu MX: Spontaneous rupture of adult Wilms' tumor: A case report and review of the literature. Can Urol Assoc J 9: E531-E534, 2015.

4. Varma AV, Malukani K, Rihal P and Nandedkar SS: Adult Wilms' tumor: A case report with review of literature. J Cancer Res Ther 11: 934-936, 2015.

5. Thevendran G, Farne HA and Kaisary AV: Wilms' tumor in a 37-yeal-old, J Clin Med Res. 2: 194-197, 2010.

6. Morabito V, Guglielmo N, Melandro F, Mazzesi G, Alesini F, Bosco S and Berloco PB: Adult Wilms tumor: Case report. Int J Surg Case Rep 6: 273-276, 2015.

7. Patnayak R, Rambabu DV, Jena A, Vijaylaxmi B, et al: Rare case of blastemal predominant adult Wilms' tumor with skeletal metastasis case report and brief review of literature. Indian J Urol 28: 447-449, 2012.

8. The reference of case 6: Guo A, Wei L, Song X and Liu A: Adult wilms tumor with intracaval and intracardiac extension: report of a case and review of literature. J Cancer 2: 132-135, 2011.

9. Masuda H, Azuma H, Nakajima F, Watsuji T, Katsuoka Y: Adult Wilms' tumor with calcification untreated for 5 years-a case report. BMC Urol 4: 5, 2004.

10. Seifert RP, McNab P, Sexton WJ, Sawczyn KK, Smith P, Coppola D and Bui MM: Rhabdomyomatous differentiation in Wilms tumor pulmonary metastases: a case report and literature review. Ann Clin Lab Sci 42: 409-416, 2012.

11. Huszno J, Starzyczny-Słota D, Jaworska M and Nowara E: Adult Wilms' tumor-diagnosis and current therapy. Cent European J Urol 66: 39-44, 2013.

12. PDQ Pediatric Treatment Editorial Board: Wilms' tumor and other childhood kidney tumors treatment (PDQ $®$ ). Bethesda MD, 2002.

13. Ali EM and Elnashar AT: Adult Wilms' tumor: Review of literature. J Oncol Pharm Pract 18: 148-151, 2012.

14. Yadav SC, Sathe PA, Ghodke RK and Fernandes GC: Giant untreated Wilms' tumor with intracardiac extension: A rare case. Indian J Pathol Microbiol 56: 68-69, 2013.

15. Wu J, Zhu Q, Zhu W and Chen W: CT and MRI imaging features and long-term follow-up of adult Wilms' tumor. Acta Radiol, Oct 8, 2015 (Epub ahead of print).

16. Shukrun R, Pode-Shakked N, Pleniceanu O, Omer D, Vax E, Peer E, Pri-Chen S, Jacob J, Hu Q, Harari-Steinberg O, et al: Wilms' tumor blastemal stem cells dedifferentiate to propagate the tumor bulk. Stem Cell Reports 3: 24-33, 2014.

17. Patnaik N, Mishra K, Saini P and Agarwal N: Primitive neuroectodermal tumor of the kidney in a young male: Case report and review of literature. Urol Ann 7: 236-239, 2015.

18. Hussong JW, Perkins SL, Huff V, McDonald JM, Pysher TJ, Beckwith JB and Coffin CM: Familial Wilms' tumor with neural elements: Characterization by histology, immunohistochemistry and genetic analysis. Pediatr Dev Pathol 3: 561-567, 2000.

19. Sircar K, Rao P, Jonasch E, Monzon FA and Tamboli P: Contemporary approach to diagnosis and classification of renal cell carcinoma with mixed histologic features. Chin J Cancer 32: 303-311, 2013.

20. Ehrlich PF, Anderson JR, Ritchey ML, et al: Clinicopathologic findings predictive of relapse in children with stage III favorable-histology Wilms tumor. J Clin Oncol 31: 1196-1201, 2013.

21. Kohler B, Biebermann H, Friedsam V, Gellermann J, Maier RF, Pohl M, Wieacker P, Hiort O, Grüters A and Krude H: Analysis of the Wilms' tumor suppressor gene (WT1) in patients 46,XY disorders of sex development. J Clin Endocrinol Metab 96: E1131-E1136, 2011.

22. Akpa MM, Iglesias DM, Chu LL, Cybulsky M, Bravi C and Goodyer PR: Wilms' tumor suppressor, WT1, suppresses epigenetic silencing of the $\beta$-catenin gene. J Biol Chem 290: 2279-2288, 2015

23. Wagner KD, Cherfils-Vicini J, Hosen N, Hohenstein P, Gilson E, Hastie ND, Michiels JF and Wagner N: The Wilms' tumour suppressor Wt1 is a major regulator of tumour angiogenesis and progression. Nat Commun 5: 5852, 2014.

24. Park M, Choi Y, Choi H and Roh J: Wilms' tumor suppressor gene (WT1) suppresses apoptosis by transcriptionally downregulating BAX expression in immature rat granulosa cells. J Ovarian Res 7: 118, 2014.

25. Provenzi M, Saettini F, Conter V, Chinaglia D, Vai P, Bruno A, Cavalleri L, Foglia C, Giraldi E, Collini P and Spreafico F: Is there a role for FDG-PET for the assessment of treatment efficacy in Wilms' tumor? A case report and literature review. Pediatr Hematol Oncol 30: 633-639, 2013. 